\title{
Knowledge and awareness of iodine nutrition among women of childbearing age
}

\author{
S.M. O’Kane, L.K. Pourshahidi, K.M. Farren, M.S. Mulhern, J.J. Strain and A.J. Yeates \\ Northern Ireland Centre for Food and Health (NICHE), University of Ulster, Cromore Road, Coleraine, \\ Co. Londonderry, BT52 1SA, UK
}

Iodine is an essential trace element necessary for the synthesis of thyroid hormones which are critical for human growth and development $^{(1,2)}$. Iodine is particularly important during pregnancy and infancy for brain and neurological development ${ }^{(3)}$. Therefore, women of child-bearing age are particularly vulnerable to the effects of iodine deficiency. Concern has been expressed in the last decade regarding the level of iodine deficiency among women of childbearing age from UK and Irish populations ${ }^{(4,5)}$. To date, no research has explored the levels of iodine knowledge in such populations. Therefore, the aim of the current study was to investigate iodine knowledge and awareness among women of childbearing age.

A 30-item questionnaire was administered online using SurveyMonkey ${ }^{\circledR}$ between February and March 2015. Females (aged 18-45 years) were asked to provide general demographic information, before the awareness of iodine and knowledge on the functions and sources of iodine were assessed. Respondents were also asked to identify the health problems resulting from iodine deficiency from a pre-defined list. Lastly, the questionnaire collected information on if and from where respondents received information on iodine and health.

To date, a total of 431 respondents have completed the questionnaire (52\% 18-25 years; $8 \%$ current smokers; $4 \%$ pregnant; $1 \%$ breastfeeding). Over half of the respondents were either not aware (19\%) or were only vaguely aware (37\%) of iodine. Those educated to higher level (BSc, MSc or PhD) had a greater awareness of the health effects of iodine, compared to those educated to secondary level (GCSE or A-Level) ( $25 \%$ vs. $13 \% ; P=0.006)$. Only $27 \%$ were aware that iodine deficiency is a current public health concern in the UK and Ireland; $22 \%$ did not think that iodine deficiency was of current concern and $51 \%$ did not know. Respondents aged 18 25 years had a greater awareness of iodine deficiency as a public health concern than those aged $26-45$ years $(17 \%$ vs. $10 \%$; $P=$ 0.037). One third of respondents (32\%) correctly identified pregnancy as the being the most important stage of the lifecycle for iodine sufficiency, $19 \%$ selected infancy and childhood and 32\% did not know at which stage of the lifecycle iodine sufficiency was most important. Only half of respondents $(48 \%)$ correctly identified fish and milk as sources of iodine. Respondents' knowledge of the health problems related to iodine deficiency was poor: $41 \%$ did not know or selected incorrect answers. Younger respondents had a significantly higher knowledge of the health problems resulting from iodine deficiency $(P<0.001)$. Less than $10 \%$ of respondents stated that they had previously received information related to iodine and health. This information was primarily given during education $(71 \%)$. Other sources of iodine nutrition information included health care professionals $(19 \%)$, media outlets $(5 \%)$ or friends and family ( $5 \%$ ). Iodine containing supplements were only consumed by $3 \%$ of respondents, and of these, $36 \%$ had been taking the supplement for less than 3 months.

The present study demonstrates that awareness of iodine and iodine deficiency is low among women of childbearing age, and may have resulted in limited use of dietary supplements in this group. There is also a large deficit in the knowledge of dietary sources and functions of iodine among a group particularly vulnerable to the effects of iodine deficiency. Few respondents had previously received information on iodine nutrition and health and may explain the low level of iodine knowledge evident from these findings. Initiatives to increase awareness and knowledge of iodine, particularly among lower-educated women, are warranted.

Ethical approval was granted from the Ulster University, School of Biomedical Sciences Ethics Filter Committee and the study was conducted according to the guidelines laid down in the Declaration of Helsinki.

1. Halpern (1991) Brain 114(2), 825-841.

2. Kapil (2007) Sultan Qaboos Univ Med J. 7(3), 267-272.

3. Zimmermann (2009) Endocr Rev. 30(4), 376-408.

4. Nawoor et al. (2006) Irish J Med Sci. 175(2), 21-24.

5. Lampropoulou et al. (2012) Proc Nutr Soc. 71(OCE2) E143. 